\title{
Stabilization of systems with changing dynamics by means of switching*
}

\author{
Miloš Žefran and Joel W. Burdick \\ Department of Mechanical Engineering, MC 104-44 \\ California Institute of Technology, Pasadena, CA 91125
}

\begin{abstract}
We present a framework for designing stable control schemes for systems whose dynamics change. The idea is to develop a controller for each of the regions defined by different dynamic characteristics and design a switching scheme that guarantees the stability of the overall system. We derive sufficient conditions for the stability of the switching scheme for systems evolving on a sequence of embedded manifolds. An important feature of the proposed framework is that if the conditions are satisfied by pairs of controllers adjacent in the hierarchy, the overall system will be stable. This makes the application of our results particularly straight forward. The methodology is applied to stabilization of a shimmying wheel, where changes in the dynamic behavior are due to switches between sliding and rolling.
\end{abstract}

\section{Introduction}

In a typical robotic application, a subsystem performing reasoning at a higher, symbolic level, interacts with a dynamical system executing continuous control laws at the lower level. But systems that combine discrete and continuous behaviors are not limited to robotics and can be found in applications ranging from manufacturing to air traffic control. Because of their dual nature, such systems are called hybrid systems. Design of discrete and continuous levels for hybrid systems is usually performed separately and their interaction is largely left to the ingenuity of the engineers. Such approach is clearly insufficient and the increasing complexity of hybrid systems calls for rigorous tools for their design, analysis and verification.

Prior work on hybrid controller design has often been limited to specific applications. Lygeros et al. [1] proposed a game-theoretic framework for design of controllers for intelligent highway systems and air traffic control systems. Puri [2] and Deshpande [3] developed methods for controller design using a simplified

*This research was supported by NSF grant CISE 9704702 and AFOSR MURI grant. version of hybrid automata. Kohn et al. developed a methodology for coordination of multiple agents [4]. Branicky \& Mitter [5] and Žefran et al. [6] employed optimal control for synthesis of open-loop trajectories. Kolmanovsky \& McClamroch [7] proposed a hybrid controller for so called cascade systems. Goodwine \& Burdick [8] developed a controllability test and a planning method for a class of hybrid systems called stratified systems.

A number of authors considered stability of hybrid controllers. Branicky [9] devised sufficient conditions for stability of a system that switches between different controllers that stabilize an equilibrium point. Based on this work, Malmborg et al. [10] proposed a strategy for choosing a controller among several available controllers so that the overall system is stable. Both papers allow dynamic equations to change, but they are primarily concerned with the case when the equilibrium point is the same for each controller so there is no need to actively drive the system into some designated region, as we do in the present paper. Stability of hybrid systems is also discussed in $[11,12]$.

The idea of driving the system through a sequence of equilibrium points until a desired equilibrium point is reached was employed in [13]. In this work, the switch between different controllers always occurs at an equilibrium point. The authors also assume that the region of attraction of each controller is known so there is no need for Lyapunov functions to prove the stability.

In this paper, we are interested in systems that change their dynamics as they move. We study the case when the changing dynamics partitions the state space into a set of embedded manifolds. Our aim is to devise a scheme for terminal control of such systems: we wish to stabilize an equilibrium point that lies on one of the sub-manifolds while descending through the sequence of manifolds in which this sub-manifold is contained. We do so by designing a hierarchy of controllers on each of the manifolds and show that the stability of the overall system can be inferred by examining the behavior of pairs of controllers that are adja- 
cent in the hierarchy. The main tool for this analysis are Lyapunov functions. The strength of our scheme is that at each step we only analyze two different controllers and the stability of the overall system automatically follows.

\section{Theoretical results}

To motivate the theoretical development we start with an example. The system that we study is the classical shimmying wheel, described in [14] and [15].
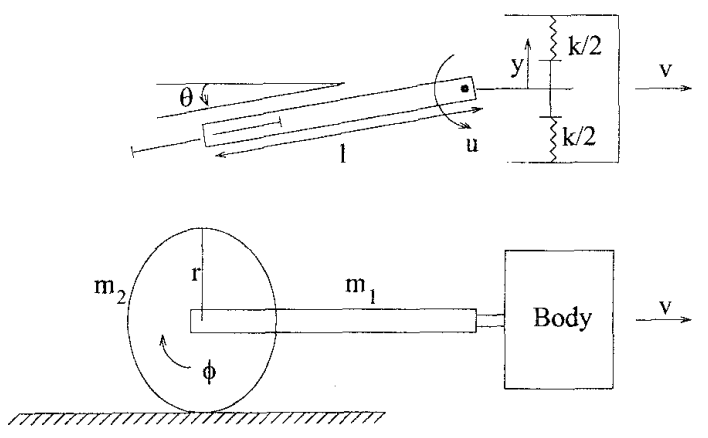

Figure 1: Schematic of a shimmying wheel.

A schematic of the shimmying wheel is shown in Fig. 1. A rigid link with a wheel is attached to a hinge joint, which is subsequently rigidly connected to a rigid object through a sliding joint between two springs (Fig. 1). The control input is the torque at the hinge joint. The object moves with a constant velocity $v$ in the direction perpendicular to the axis of the sliding joint. The shimmying wheel can be seen as a simplified model of wheeled systems such as a robotic vehicle towing a trailer, an aircraft nose wheel or a motorcycle front wheel [15].

The goal of the control is to stabilize the wheel so that the bar is aligned with the direction of $v$ (perpendicular to the sliding axis) and the slider is in the neutral position between the two springs (the forces of the springs are equal in magnitude and of the opposite sign). This task is complicated by the fact that the system can operate in two regimes: the wheel can either roll without sliding or it can slip. The system will switch between rolling and sliding depending on the magnitude of the contact force between the wheel and the ground: the wheel will slip if the force in rolling would be greater than the friction force. If we assume a feedback control law for the torque about the hinge joint, the contact force is completely determined by the state of the system and the state space gets divided into two regions separated by a switching surface on which the contact force equals the friction force. In each of the regions the equations of motion are different. It is therefore unlikely that a single controller could stabilize the system and even if one exists it is not clear how to design it.

We continue by stating the problem in a more formal way. Suppose we have a dynamical system $\Sigma$ and a sequence of (differentiable, connected) manifolds $\mathbb{R}^{N} \supseteq M_{1} \supseteq M_{2} \supseteq \ldots \supseteq M_{n}$. An example of such sequence is shown in Fig. 2. On each manifold, the system is described with a different set of equations:

$$
\dot{x}_{i}=f_{i}\left(x_{i}, u_{i}, t\right)
$$

where $x_{i}$ is the state of the system and $u_{i}$ is the vector of inputs for the system evolving on the submanifold $M_{i}$. Note that the dimensions of the manifolds might be different. In the case of the shimmying wheel, the manifolds $M_{1}$ and $M_{2}$ would correspond to sliding and rolling, respectively, where $M_{1}$ is the whole space and $M_{2}$ is the subspace on which the rolling constraint is satisfied.

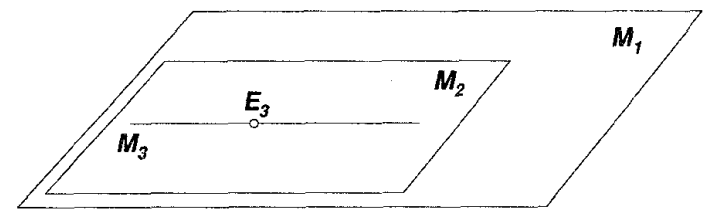

Figure 2: A sequence of embedded manifolds.

Let $E_{n} \subseteq M_{n}$ be a manifold to which we wish to steer the system $\Sigma$. The problem that we address in this paper is how to design a sequence of feedback controllers:

$$
u_{i}=g_{i}\left(x_{i}, t\right) \text {, }
$$

and a switching scheme that (if possible globally) stabilizes the system to $E_{n}$. This task is complicated by the fact that, in general, we can not guarantee that the system will stay on a manifold $M_{i}$ once it starts evolving on it; it is possible that it gets pushed back to the manifold $M_{i-1}$. This is for example the case with the shimmying wheel: a disturbance can always cause the rolling wheel to slip.

\subsection{Sufficient conditions for stability}

Because of the limitations on space we state the results in this section without a proof. The interested reader is referred to [16] for details. We also note that the conventional Lyapunov theory has to be appropriately modified to study stabilization of manifolds as opposed to single points. The reader is referred to $[17,18]$ for further details. Here we just note that a distance between a point $x$ and a set $E \subseteq \mathbb{R}^{n}$ is defined as usual by $\rho(x, E)=\inf _{y \in E} d(x, y)$ and that a ball with radius $R$ around $E$ is the set $B(E, R)=\{x \mid \rho(x, E)<R\}$. 
Take a control system $\Sigma$ evolving on two manifolds $M_{1} \supseteq M_{2}$. Let $g_{1}$ be a feedback controller on $M_{1}$ (i.e., $\left.u_{1}=g_{1}(x, t)\right)$ that steers the system to a manifold $E_{1}$ and $g_{2}$ a feedback controller on $M_{2}$ that steers the system to a manifold $E_{2}$. In order to steer an arbitrary trajectory to a submanifold of $M_{2}$, we must require that $E_{1} \subseteq M_{2}$, otherwise the system might get stuck on $E_{1}$ without being able to switch to the controller $g_{2}$. Assume we can construct a Lyapunov function $V_{2}$ that shows that $g_{2}$ stabilizes $E_{2}$. Let

$$
\begin{aligned}
\mathcal{S}: \quad \mathbb{R}^{n} \times\{1,2\} & \rightarrow\{1,2\} \\
(x, \eta) & \mapsto \mathcal{S}(x, \eta)
\end{aligned}
$$

denote the switching scheme. In other words, the function $\mathcal{S}$ selects the controller to be used, depending on the state $x$, and the controller that is currently used, $\eta$. Clearly, $\mathcal{S}(x, \eta)=2$ implies $x \in M_{2}$, since $g_{2}$ is only defined on $M_{2}$. Let the sequence $t_{1}<t_{2}<\ldots$ describe the switches between the controllers $g_{1}$ and $g_{2}$, so that the switch occurs from $g_{1}$ to $g_{2}$ for times with odd indices $\left(t_{2 k+1}\right)$ and from $g_{2}$ to $g_{1}$ for times with even indices $\left(t_{2 k}\right)$ (Figure 3 ). The following lemma then gives sufficient conditions for $E_{2}$ to be globally attractive:

Lemma 2.1 Let the switching scheme $\mathcal{S}$ satisfy the following conditions:

1. For every trajectory $x(t)$ and any time $T$, there exists $t_{s}>T$ such that $\mathcal{S}\left(x\left(t_{s}\right), \eta\left(t_{s}\right)\right)=2$.

2. $V_{2}\left(t_{2 k}\right) \geq V_{2}\left(t_{2 k+1}\right)$ for every positive integer $k$.

3. There exists a $\Delta$ such that $t_{2 k}-t_{2 k-1}>\Delta$ for every positive.integer $k$.

4. There exists $L>0$ such that $S(x, 2)=2$ for every $x \in B\left(E_{2}, L\right) \cap M_{2}$.

Then the submanifold $E_{2}$ is globally attractive.

Lemma 2.1 allows us to prove the following theorem:

Theorem 2.2 Let $\Sigma$ be a control system evolving on the chain of manifolds $M_{1} \supseteq M_{2} \supseteq \ldots \supseteq M_{n}$. Let $g_{i}, E_{i}, V_{i}$ and $\mathcal{S}_{i}$ be the feedback controller, the attractive manifold for the system under the control of $g_{i}$, the corresponding Lyapunov function on $M_{i}$ and the switching scheme that determines when we switch from $g_{i}$ to $g_{i+1}$. If for each $i<n$, the triples $\left(M_{i}, g_{i}, E_{i}\right)$ and $\left(M_{i+1}, g_{i+1}, E_{i+1}\right)$, together with the Lyapunov function $V_{i+1}$ defined on $M_{i+1}$ and the switching scheme $\mathcal{S}_{i}$ satisfy the conditions of Lemma 2.1, then the system will be steered to $E_{n}$ under the switching control:

$$
\mathcal{S}(x, \eta)= \begin{cases}\mathcal{S}_{n-1}(x, \eta) & \eta \in\{n-1, n\}, x \in M_{n-1} \\ \ldots & \\ \mathcal{S}_{i}(x, \eta) & \eta \in\{i, i+1\}, x \in M_{i} \backslash M_{i+1} \\ \ldots & \\ \mathcal{S}_{1}(x, \eta) & \eta \in\{1,2\}, x \in M_{1} \backslash M_{2}\end{cases}
$$

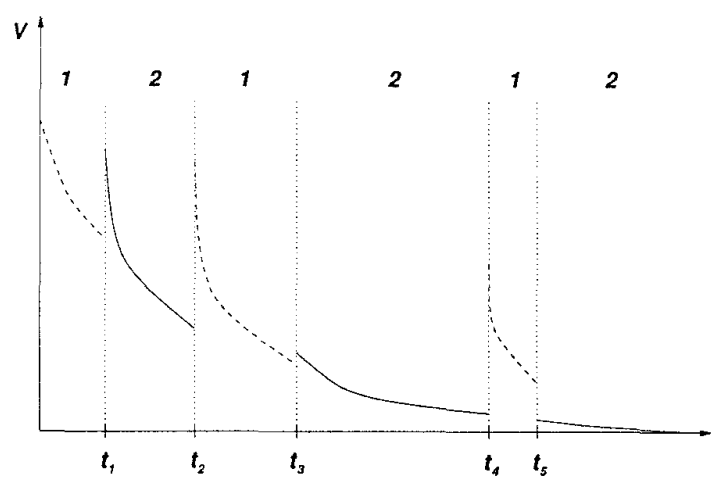

Figure 3: The value of the Lyapunov function as the system switches.

\section{Example}

The above results provide a framework for designing hybrid control schemes. We now show by example how to apply this methodology. We again consider the shimmying wheel (Fig. 1). If $F=\left\{F_{x}, F_{y}\right\}^{T}$ is the reaction force of the ground on the wheel, the equations of motion for the system are:

$$
H\left[\begin{array}{c}
\ddot{y} \\
\ddot{\theta} \\
\ddot{\phi}
\end{array}\right]+\left[\begin{array}{c}
k y+\frac{l}{2}\left(m_{1}+2 m_{2}\right) \dot{\theta}^{2} \sin \theta \\
0 \\
0
\end{array}\right]=A^{T} F+\left[\begin{array}{l}
0 \\
u \\
0
\end{array}\right]
$$

where $H$ is the inertia matrix:

$H=\left[\begin{array}{ccc}m_{1}+m_{2} & -\frac{l}{2}\left(m_{1}+2 m_{2}\right) \cos \theta & 0 \\ -\frac{l}{2}\left(m_{1}+2 m_{2}\right) \cos \theta & l^{2}\left(\frac{m_{1}}{3}+m_{2}\right)+\frac{r^{2}}{4} m_{2} & 0 \\ 0 & 0 & \frac{r^{2}}{2} m_{2}\end{array}\right]$

and

$$
A=\left[\begin{array}{rrr}
0 & l \sin \theta & -r \cos \theta \\
1 & -l \cos \theta & -r \sin \theta
\end{array}\right]
$$

is the matrix that relates the relative velocity $v_{r}$ between the wheel and the ground at the contact point to the rate of change of the generalized coordinates: $v_{r}=\{v, 0\}^{T}+A\{\dot{y}, \dot{\theta}, \dot{\phi}\}^{T}$. The system has 6 states: 3 generalized coordinates and 3 generalized velocities.

When the wheel is rolling, we have an additional constraint:

$$
v_{r}=0
$$

In this case, the force $F=F_{c}$ is the constraint force that prevents slippage of the wheel and it can be eliminated from Eq. (4) using Eq. (6) [14, 15]. Since (6) represents two constraint equations, the dimension of the system in pure rolling drops to 4 .

When the wheel is sliding, we have the following expression for the reaction force $F=F_{s}$ :

$$
F_{s}=-\mu_{d} \frac{v_{r}}{\left\|v_{r}\right\|}\left(m_{1}+\frac{m_{2}}{2}\right) g
$$


where $\mu_{d}$ is the coefficient of (dynamic) friction and $g$ is the gravity constant.

The switch from rolling to sliding occurs when the amplitude of the constraint force exceeds the amplitude of the (static) friction:

$$
\left\|F_{c}\right\|>\mu_{s}\left(m_{1}+\frac{m_{2}}{2}\right) g \quad \Rightarrow \quad \text { rolling } \rightarrow \text { sliding }
$$

The condition for the switch from sliding to rolling is that the relative velocity is 0 and that the amplitude of the frictional force is greater than the amplitude of the constraint force:

$$
v_{r}=0 \&\left\|F_{c}\right\| \leq \mu_{s}\left(m_{1}+\frac{m_{2}}{2}\right) g \quad \Rightarrow \quad \text { sliding } \rightarrow \text { rolling }
$$

To avoid problems with the uniqueness of solutions to the dynamic equations, we require that $\mu_{d}<\mu_{s}$.

The analysis of the system can be simplified by observing that $\phi$ does not occur in any of the equations. It is therefore a cyclic variable and we can limit our study to the dynamics of $y$ and $\theta$. In the formalism of Section 2, the reduced system thus evolves on manifolds $M_{1}$ and $M_{2}$ of dimension 4 and 3 , respectively, where $M_{1}=\mathbb{R}^{4}$ and $M_{2}$ is defined by Eq. (6).

The goal of the control is to stabilize the wheel to the state $y=0$ and $\theta=0$. To achieve this goal we will design three controllers: a controller $g_{1}$ for the system in sliding regime (defined on $M_{1}$ ) and controllers $g_{2}$ and $g_{3}$ for the system in the rolling mode (defined on $M_{2}$ ). To apply Theorem 2.2 we introduce an additional manifold $M_{3}=M_{2}$. The idea is to steer the system with the controllers $g_{1}$ and $g_{2}$ to a state from which we can stabilize the system to a desired point with the controller $g_{3}$.

To design a controller for the system evolving on $M_{1}$, we observe that the control input can linearize the dynamic response for $\theta$. Therefore, $\theta$ can be made to exponentially converge to 0 . A short calculation shows that for $\theta=\dot{\theta}=0$, the dynamics for $\phi$ is given by:

$$
\ddot{\phi}=-\frac{\mu_{d}\left(m_{1}+2 m_{2}\right) g(r \dot{\phi}-v)}{m_{2} r \sqrt{\dot{y}^{2}+(r \dot{\phi}-v)^{2}}}
$$

so $\dot{\phi}$ asymptotically converges to $\frac{v}{r}$. Similarly, the dynamics for $y$ at $\theta=\dot{\theta}=0$ equals:

$$
\ddot{y}=-\frac{\mu_{d}\left(m_{1}+2 m_{2}\right) g \dot{y}}{2\left(m_{1}+m_{2}\right) \sqrt{\dot{y}^{2}+(r \dot{\phi}-v)^{2}}}-\frac{k y}{2\left(m_{1}+m_{2}\right)}
$$

The limit of the first term on the right side is not well defined as $v_{r} \rightarrow 0$, but further analysis shows that the system will converge to $\dot{y}=0$ and in addition $|y|<\frac{\mu_{d}\left(\frac{m_{1}}{2}+m_{2}\right) g}{k}$. We therefore conclude that in the reduced space $(y, \theta, \dot{y}, \dot{\theta})$ with the cyclic variable $\phi$ eliminated, $E_{1}$ is the line segment $(y, 0,0,0)$, where $|y| \leq \frac{\mu_{d}\left(m_{1}+2 m_{2}\right) g}{2 k}$.

To design the controller $g_{2}$ on $M_{2}$ (only defined in the rolling mode), we use the same idea as above. We first have to eliminate the constraint force using Eq. (6), after which we can compute $u$ that cancels the nonlinearities in the equation for $\ddot{\theta}$. Since the system is in the rolling mode, the constraint (6) also implies that the system will simultaneously approach $\dot{y}=0$ and $\dot{\phi}=\frac{v}{r}$. However, $y$ is not forced to 0 and can have an arbitrary value. In this case, the attractive manifold $E_{2}$ is therefore given (in the reduced space) by a line $(y, \theta, \dot{y}, \dot{\theta})=(y, 0,0,0)$. We will also need a Lyapunov function $V_{2}$ that assures stability of $E_{2}$. Since the dynamics of the system is given completely by the dynamics of $\theta$, it suffices to construct a Lyapunov function that shows the asymptotic stability of a linear dynamic equation for $\theta$, which can be easily. done.

Finally, we derive a controller $g_{3}$ on $M_{2}$ that stabilizes the reduced system to the point $E_{3}=$ $(y, \theta, \dot{y}, \dot{\theta})=(0,0,0,0)$. To this end, we investigate the dynamics for $y$ with the constraint force eliminated. We could see that we can again cancel the nonlinear terms in the equation for $\ddot{y}$ and make the point $(y, \dot{y})=(0,0)$ asymptotically stable. A short calculation shows that at this point, the dynamics for $\theta$ becomes:

$$
\dot{\theta}=\frac{v}{l} \sin \theta
$$

so that $\theta=0$ is a stable equilibrium point everywhere except for $\theta=\pi$. This, together with the constraint equation (6), also implies that the system will converge to $\dot{\phi}=\frac{v}{r}$.

We construct the Lyapunov function $V_{3}$ in two steps. We want the dynamics of $y$ to be dominant until $y$ almost converges to the equilibrium point $y=0, \dot{y}=0$, where we want the (stable) dynamics for $\theta$ to take over. Let $V_{3}^{y}$ be the Lyapunov function for the stable linear equation for $y$. It is easy to see that:

$$
V_{2}^{\theta}=\theta^{2}
$$

is a Lyapunov function for the system (10). Now consider the function:

$$
V_{2}=C_{1} V_{2}^{y}+e^{-C_{2}\left(y^{2}+\dot{y}^{2}\right)} V_{2}^{\theta} .
$$

The function is positive definite and we can choose the constants $C_{1}$ and $C_{2}$ to obtain a negative derivative along the trajectories.

Next, we have to design the switching schemes. The switching scheme $\mathcal{S}_{1}$ is quite simple:

$$
\mathcal{S}_{1}(x, \eta)= \begin{cases}2 & x \in M_{2},\left\|F_{c}\right\| \leq \frac{\mu_{d}}{2}\left(m_{1}+2 m_{2}\right) g \\ 1 & \text { otherwise }\end{cases}
$$



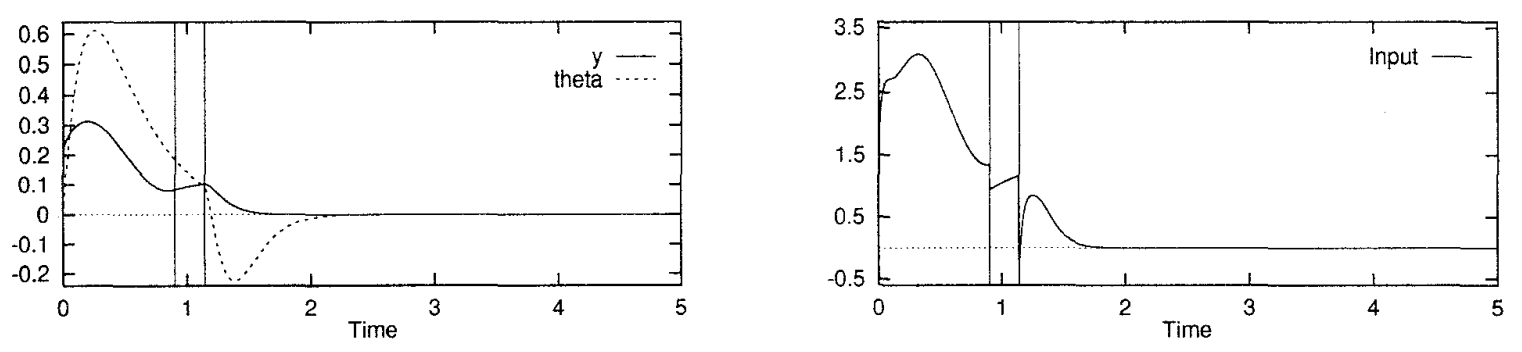

Figure 4: A typical simulation run.

The controller $g_{2}$ has a singularity at $\theta= \pm \frac{\pi}{2}$, but on these two hyperplanes the constraint force is unbounded, which means that they do not intersect (the closure of) $\mathrm{M}_{2}$.

The switching scheme $\mathcal{S}_{2}$ is defined in the following way:

$$
\mathcal{S}_{2}(x, \eta)=\left\{\begin{array}{c}
3 \eta=2, x \in B\left(E_{3}, R_{\text {in }}\right), V_{3}(x) \leq V_{3}^{3 \rightarrow 2}, \\
\quad\left\|F_{c}\right\| \leq \frac{\mu_{s}}{4}\left(m_{1}+2 m_{2}\right) g \\
3 \quad \eta=3, x \in B\left(E_{3}, R_{\text {out }}\right) \\
2 \text { otherwise }
\end{array}\right.
$$

where $R_{\text {in }}<R_{\text {out }}<\frac{\pi}{2}$ (this guarantees that $B\left(E_{3}, R_{\text {out }}\right)$ does not intersect the hyperplanes $\theta=$ $\left.\pm \frac{\pi}{2}\right)$, and $V_{3}^{3 \rightarrow 2}$ is the value of $V_{3}$ when the system last switched from the controller $g_{3}$ to the controller $g_{2}$. Again, we avoid the hyperplanes $\theta= \pm \frac{\pi}{2}$ because $g_{3}$ becomes singular there. Observe that the switching scheme explicitly encodes condition (2) of Lemma 2.1. The other restrictions in the switching scheme are necessary to satisfy condition (3).

The next step would be to check that the conditions of Theorem 2.2 are satisfied. According to the theorem, it suffices to show that $g_{1}$ and $g_{2}$ stabilize $E_{2}$, and that $g_{2}$ and $g_{3}$ stabilize $E_{3}$. In the interest of keeping the presentation short, the proofs will be omitted but we refer the interested reader to [16] for the details. Here we only mention that in order to show that the controller $g_{2}$ can arbitrarily decrease the Lyapunov function $V_{3}$ so that the system can switch to $g_{3}$, we use a modified controller:

$$
\hat{g}_{2}(x)=\left(1-c_{1} e^{-c_{2} V_{2}(x)}\right) g_{2}(x)+c_{1} e^{-c_{2} V_{2}(x)} g_{3}(x)
$$

This controller behaves as $g_{2}$ away from $E_{2}$ and as $g_{3}$ close to $E_{2}$. It will therefore bring the system towards $E_{2}$ and once close to $E_{2}$ cause the Lyapunov function $V_{3}$ to decrease.

\subsection{Simulation results}

A typical simulation run of the system controlled with the derived controllers is shown in Fig. 4. The system starts in the sliding regime with the controller $g_{1}$ active. At $0.9 \mathrm{~s}$ the wheel stops sliding and the controller $g_{2}$ takes over. At $1.14 \mathrm{~s}$ the system switches again, this time to the controller $g_{3}$ that stabilizes the system to the desired state. The switches between different controllers cause discontinuities of the input, as Fig. 4.b. shows. It can be seen in Fig. 4.a that while the controllers $g_{1}$ and $g_{2}$ are active, $\theta$ is the controlled variable and it decreases to 0 . When the controller $g_{3}$ becomes active, the controlled variable becomes $y$ (so it decreases to 0 ) and $|\theta|$ initially increases. After $y$ becomes small, $|\theta|$ also decreases to 0 .

The next figure illustrates that the modified controller $\hat{g}_{2}$ decreases the Lyapunov function $V_{3}$. Variables $y$ and $\theta$ are shown in Fig. 5.a, while the Lyapunov functions $V_{2}$ and $V_{3}$ are shown in Fig. 5.b. The system starts in the rolling regime with the controller $g_{3}$ active, however during the first $0.1 \mathrm{~s}$ it switches first to the controller $g_{2}$ and then to the sliding regime and the controller $g_{1}$ (these switches are not shown). At the switch from $g_{3}$ to $g_{2}$ the value of the Lyapunov function $V_{3}$ us 263.4. To show that the controller can arbitrary decrease $V_{3}$, we modified the switching scheme $\mathcal{S}_{2}$ so that the value of the Lyapunov function $V_{3}$ at the switch from $g_{2}$ to $g_{3}$ has to be half the value of the function at the switch from $g_{3}$ to $g_{2}$. In our case, the function $V_{3}$ therefore has to decrease to 131.7 in order to switch to the controller $g_{3}$. At the time $0.38 \mathrm{~s}$, the system switches from sliding to rolling and to the controller $g_{2}$. The controller decreases the Lyapunov function until it reaches the desired value at the time $1.30 \mathrm{~s}$ when the system switches to the controller $g_{3}$ and the system is stabilized. Figure 5.a also shows that the controller $g_{2}$ does not drive $\theta$ to 0 but to some offset value that guarantees the decreasing of $V_{3}$.

\section{Conclusion}

We investigated the problem of stabilizing a system with changing dynamics with a sequence of controllers. We studied the case when the system evolves on a sequence of embedded manifolds and derived sufficient conditions under which the switching scheme employing different controllers can be guaranteed to stabilize the system to the desired manifold. These sufficient conditions give direct guidance for the design of appropriate controllers. The results were applied to the stabilization of the shimmying wheel. We were able 

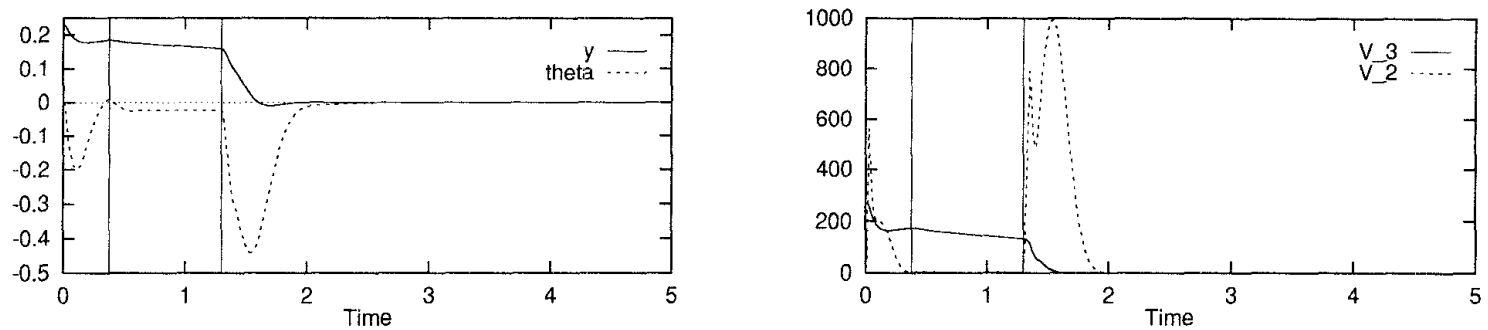

Figure 5: A modified controller guarantees decreasing of $V_{3}$.

to design a switching scheme that provably stabilizes this system.

The described work can be extended in several directions. An immediate extension would be to consider less restrictive topology, where the manifolds form a more general structure, not necessarily a sequence. An important open question is also how to stabilize periodic orbits in problems such as walking.

\section{References}

[1] J. Lygeros, D. N. Godbole, and S. S. Sastry, "A game theoretic approach to hybrid system design," in LNCS 1066, pp. 1-12, Springer-Verlag, 1996.

[2] A. Puri, Theory of hybrid systems and discrete event systems. PhD thesis, U. C. Berkeley, 1995.

[3] A. Deshpande and P. Varaiya, "Viable control of hybrid systems," in LNCS 999, pp. 128-147, Springer-Verlag, 1995.

[4] W. Kohn, A. Nerode, J. B. Remmel, and X. Ge, "Multiple agent hybrid control: carrier manifolds and chattering approximations to optimal control," in Proceedings of the 33rd IEEE Conference on Decision and Control, (Lake Buena Vista, FL), pp. 4221-4227, 1994.

[5] M. S. Branicky and S. K. Mitter, "Algorithms for optimal hybrid control," in Proceedings of the 34th IEEE Conference on Decision and Control, (New Orleans, LA), pp. 2661-2666, 1995.

[6] M. Žefran, J. Desai, and V. Kumar, "Continuous motion plans for robotic systems with changing dynamic behavior," in Robotic motion and manipulation, pp. 113-128, Wellesley, MA: A. K Peters, 1997.

[7] I. Kolmanovsky and H. N. McClamroch, "Hybrid feedback laws for a class of cascade nonlinear control systems," IEEE Trans. on Automatic Control, vol. 41, no. 9, pp. 1271-1282, 1996.
[8] B. Goodwine and J. W. Burdick, "A general method for motion planning for quasi-static legged robotic locomotion." Preprint, 1997.

[9] M. S. Branicky, "Stability of switched and hybrid systems," in Proceedings of the 33rd IEEE Conference on Decision and Control, (Lake Buena Vista, FL), pp. 3498-3503, 1994.

[10] J. Malmborg, B. M. Bernhardsson, and K. J. Aström, "A stabilizing switching scheme for multi-controller systems," in 13th IFAC World Congress, (San Francisco, CA), 1996.

[11] P. Peleties and R. DeCarlo, "Asymptotic stability of m-switched systems using Lyapunov-like functions," in American Control Conf., (Boston), pp. 1679-1684, 1991.

[12] L. Hou and A. N. Michel, "Stability analysis of a general class of hybrid dynamical systems," in Proceedings of the $1997 \mathrm{ACC}$, (Albuquerque, NM), pp. 2805-2809, 1997.

[13] R. R. Burridge, A. A. Rizzi, and D. E. Koditschek, "Sequential composition of dynamically dexterous robot behaviors." Preprint, 1996.

[14] G. Stépán, "Chaotic motion of wheels," Vehicle System Dynamics, vol. 20, pp. 341--351, 1991.

[15] B. Goodwine and G. Stépán, "Controlling unstable rolling phenomena." To appear in the Journal of Vibration and Control, 1997.

[16] M. Žefran and J. W. Burdick, "Switching control on embedded manifolds," tech. rep., Caltech, 1997.

[17] W. Hahn, Stability of motion. Springer-Verlag, 1967.

[18] A. Vannelli and M. Vidyasagar," "Theory of partial stability theorems, converse theorems, and maximal Lyapunov functions," in Proc. Annu. Southeast Symp. Syst. Theory, (Piscataway, NJ), pp. 16-20, 1980. 